\title{
Severe acute respiratory syndrome coronavirus 2 (SARS-CoV-2) and the neuroendocrine stress axis
}

\author{
Charlotte Steenblock $\mathbb{D}^{1} \cdot$ Vladimir Todorov $^{1} \cdot$ Waldemar Kanczkowski ${ }^{1} \cdot$ Graeme Eisenhofer $^{1,2} \cdot$ Andreas SchedI $^{3}$. \\ Ma-Li Wong $\mathbb{1}^{4} \cdot$ Julio Licinio ${ }^{4}$ Michael Bauer $\mathbb{D}^{5} \cdot$ Allan H. Young ${ }^{6} \cdot$ Raul R. Gainetdinov ${ }^{7,8} \cdot$ Stefan R. Bornstein ${ }^{1,9}$
}

Received: 21 April 2020 / Revised: 23 April 2020 / Accepted: 23 April 2020 / Published online: 7 May 2020

(c) Springer Nature Limited 2020

Severe acute respiratory syndrome coronavirus 2 (SARS-CoV2 ), first identified in December 2019, causes coronavirus disease 2019 (COVID-19). It is reasonable to presume that SARS-CoV-2 infection will cause activation of the endocrine stress axis at several levels. The endocrine stress system involves communication among different tissues, including among others, the brain and components of the hypothalamicpituitary-adrenal (HPA) axis. Dysregulation of the stress system leads to morphologic changes in target organs or epigenetic stem cell changes. These in turn might contribute to the development or exacerbation of various psychiatric disorders. We discuss here the potential impact of an infection such as SARS-CoV-2 on a systemic level and reflect on its potential influence on the endocrine stress axis and the consequent long-

Charlotte Steenblock

charlotte.steenblock@uniklinikum-dresden.de

1 Department of Internal Medicine III, University Hospital Carl Gustav Carus, Technische Universität Dresden, Dresden, Germany

2 Institute of Clinical Chemistry, University Hospital Carl Gustav Carus, Technische Universität Dresden, Dresden, Germany

3 Université Côte d'Azur, INSERM, CNRS, iBV, Nice, France

4 State University of New York, Upstate Medical University, Syracuse, NY, USA

5 Department of Psychiatry and Psychotherapy, Carl Gustav Carus University Hospital, Technische Universität Dresden, Dresden, Germany

6 Centre for Affective Disorders, Department of Psychological Medicine, Institute of Psychiatry, Psychology \& Neuroscience, King's College London, London, UK

7 Institute of Translational Biomedicine, St. Petersburg State University, St. Petersburg, Russia

8 St. Petersburg State University Hospital, St. Petersburg State University, St. Petersburg, Russia

9 Diabetes and Nutritional Sciences Division, King's College London, London, UK term consequences. As SARS-CoV-2 and its host receptor, angiotensin-converting enzyme 2 (ACE2), both impact the HPA axis, we also discuss potential therapeutic strategies.

How humans cope with distinct stressors is crucial for their health as imbalance of the stress response can impact the development of disease and the course of psychiatric disorders. Among the latter, major depression, anxiety disorders, and the occupational burnout syndrome may also contribute to somatic manifestations such as inflammatory, immune, oncologic, metabolic, and cardiovascular disorders (reviewed in [1-5]).

COVID-19, caused by SARS-CoV-2, may lead to a full cascade of stress responses. This can be expected to include activation of the neuroendocrine HPA stress axis and other systems that maintain homeostasis. Foremost amongst these is the renin-angiotensin-aldosterone system (RAAS), which is directly impacted by SARS-CoV-2 [6]. Therefore, for infected patients there is need to consider potential complications or benefits for treatments that target regulatory endocrine systems, including drugs such as corticosteroids, ACE inhibitors, or angiotensin receptor blockers [7-10].

Apart from infected patients, others may also suffer the consequences of SARS-CoV-2. In addition to anxiety of getting or being infected, stress may be associated with the loss of family members or friends, patient care, or with quarantine measures. Downstream consequences, such as emotional and financial instability are also likely to impact mental health and from this also the associated somatic disorders. Therefore, numerous considerations relevant to mental health and general well-being need to be anticipated during and after a pandemic and resulting quarantine measures, such as what is occurring in COVID-19 [11].

\section{Stress response}

The ability of an organism to adapt to encountered stressors involves the sympathoadrenal system and the HPA axis, 
and interactions between these two systems play a central role in adaptation. Patients with stress-related disorders may exhibit increased vulnerability to mental illness for several years after the stress experience [12].

On a systemic level, stressors activate the sympathetic branch of the autonomic nervous system followed by activation of the HPA axis. Splanchnic nerve activation triggers chromaffin cells in the adrenal medulla to release the catecholamines, epinephrine and norepinephrine, for mediating a rapid response. When the HPA axis is activated, corticotropin-releasing hormone $(\mathrm{CRH})$ and arginine vasopressin (AVP) are secreted from the paraventricular nucleus of the hypothalamus. CRH and AVP then activate the anterior pituitary to secrete adrenocorticotropic hormone (ACTH), which in turn stimulates the adrenal cortex to produce corticosteroids [13].

Glucocorticoid receptors are expressed in several areas of the adult brain such as the hypothalamus, pituitary, hippocampus, and amygdala, which are all involved in glucocorticoid feedback. An appropriate response during acute stress is imperative for survival, but frequent or prolonged activation can change the functional tone of stress systems with ensuing deleterious effects.

Excessive or chronic activation of the endocrine stress axis, which also leads to a disrupted feedback loop, triggers and contributes to numerous human disorders and stress conditions in humans, including anxiety, post-traumatic stress disorder, major depressive disorder, and occupational burnout. In addition, gonadal steroids can differentially affect the HPA axis resulting in sex differences in responses to stress [14]. Animal models and human studies support an increased risk in males for behavioral or neurodevelopmental disorders in response to prenatal and early life stress, whereas females appear to have an increased risk in adolescence and throughout adulthood, especially if they have experienced stress-related early life adversity or trauma. During the perimenopause and menopausal transition, women are at greater risk for presenting with affective disorders including depression as well as schizophrenia [15].

\section{Infection with SARS-CoV-2}

Entry of the corona viruses SARS-CoV and SARS-CoV-2 into cells depends on binding of the viral spike (S) glycoproteins to cellular ACE2, which the virus uses as its entry receptor. Furthermore, $\mathrm{S}$ proteins are primed by host cell proteases [16, 17]. This subsequently leads to fusion of the viral envelope and the plasma membrane or the endosome membrane of the host. It has been indicated that the SARSCoV-2 S protein binds to ACE2 with 10-20-fold higher affinity than the $\mathrm{S}$ protein of SARS-CoV. This might underlie the high spread of SARS-CoV-2 [18].

ACE2 is a carboxypeptidase, which controls the generation of the vasodilating angiotensin 1-7 from angiotensin II. ACE2 can also cleave angiotensin I to angiotensin 1-9, which can then be further converted to angiotensin 1-7 by ACE. ACE2, angiotensin 1-7 and its MAS receptor constitute the vasoprotective arm of the RAAS. Excessive activation of the classical RAAS leads to arterial hypertension, accompanied by immuno-metabolic disorders and obligatory culminates in end-organ damage in the absence of adequate therapy (for recent reviews see [19-23]). The RAAS and in particular ACE2 appear to be attractive targets to fight COVID-19. Therein, the role of ACE inhibitors and AT1R blockers is currently highly controversial. On the one hand, these drugs could increase ACE2 expression and potentiate SARS-CoV-2 cell entry [24]. On the other hand, increased ACE2 production would result in a higher circulating level of soluble ACE2, which neutralizes SARSCoV-2 [25]. Yet, the precise role of the available RAAStargeting drugs in COVID-19 disease waits to be unraveled.

ACE2 has been shown to have an effect on the stress response and on anxiety. Mice overexpressing ACE2 are less anxious and have reduced plasma corticosterone and pituitary expression of proopiomelanocortin [26]. The same effect was observed when ACE2 overexpression was targeted to the CRH-synthesizing cells of the hypothalamus suggesting that especially the HPA axis is affected [27]. In mice transgenic for ACE2, the olfactory bulb of the brain is a major target organ for SARS infection leading to transneural spread [28-30].

Expression of ACE2 has been noted not only in the respiratory tract, lung, ileum, bladder, esophagus, heart, and kidney [31], but also in the the hypothalamus, pituitary and adrenal [32]. These results fit with the organ distribution of SARS-CoV observed in patients who died of SARS [33]. Intriguingly, in these patients, following the lung, the adrenal belonged to the organs with the highest concentration of virus particles. In addition, the pituitary was affected [33] making it clear that the HPA axis is targeted during a coronavirus infection. Furthermore, the gastrointestinal tract is targeted [33] suggesting associations with appearance of gastrointestinal infection symptoms in some patients [34]. As ACE2 is also expressed in the human brain this might explain why infection of the central nervous system is regularly observed in SARS patients [35, 36].

Although an equal number of COVID-19 cases between men and women has been reported, there seem to be sex differences in mortality and vulnerability to the disease [37]. Emerging evidence suggests that more men than women are dying [38, 39]. It has been speculated that this is due to sexbased immunological [40] or other differences, such as 
patterns and prevalence of smoking [41]. An alternative hypothetical explanation could involve sex-dependent differences in responsiveness of HPA and other hormonal/stress systems. Further research is needed to confirm this hypothesis.

In conclusion, the strong interaction and reciprocal communication between the organs of the endocrine stress system in a coronavirus infection require a deeper understanding of the adaptation of all components to the infection. Furthermore, it has to be considered that certain therapies impacting expression of ACE2 might also influence the RAAS and the neuroendocrine stress axis, which may lead to long-term consequences, as prolonged exposure to stressors increases the risk to develop major depressive, anxiety and post-traumatic stress disorders [42].

\section{SARS-CoV-2 antiviral defense}

Maintenance of homeostasis during immune challenges, such as a virus infection, involves activation of the immune system, resolution of the challenge, and protection of the host against potentially toxic inflammatory processes. Upon immune challenge, the immune system is activated to produce a number of cytokines. One functional group of cytokines mediates the innate immune response and includes several proinflammatory cytokines: tumor necrosis factor- $\alpha$ (TNF- $\alpha$ ), interleukin-1 (IL-1), and IL-6, and the type I interferons (IFN- $\alpha / \beta)$. These cytokines are released in the early stages of an immune response. Another group of cytokines, such as the T cell cytokines IL-2 and IFN- $\gamma$ (type II interferons), which are especially important in mediating antiviral defenses, mediates the later adaptive immunity.

In addition to contributing to the progression of the immune response against viral infection, cytokines activate the HPA axis, resulting in the release of adrenal glucocorticoids [43]. In turn, glucocorticoids exert negative feedback influences on immune cells to suppress further synthesis and release of cytokines, thereby protecting the host from the detrimental consequences of an overactive immune response (e.g., tissue damage, autoimmunity, or septic shock) [44, 45]. A number of cytokines have been measured in serum of COVID-19 patients [46]. Initial plasma IL-1B, IL-1RA, IL-7, IL-8, IL-9, IL-10, bFGF, GCSF, GMCSF, IFN- $\gamma$, IP-10, MCP1, MIP-1A, MIP-1B, PDGF, TNF- $\alpha$, and VEGF concentrations were higher in patients than in healthy adults, whereas plasma levels of IL5, IL-12p70, IL-15, Eotaxin, and RANTES were similar in patients and controls. IFN- $\alpha$ and $\beta$ were not measured. These results suggest that an overproduction of cytokines might be responsible for the damage of the lungs in COVID-19 patients. However, also host factors such as expression of ACE2 and underlying diseases such as hypertension, chronic obstructive pulmonary disease, diabetes, and cardiovascular disorders might influence susceptibility to infection and progression of the disease [34, 47].

Both SARS-CoV and Middle East respiratory syndrome (MERS)-CoV were shown to induce very little type I IFN in most cell types. This might be due to different reasons such as storage of coronaviral dsRNA in double-membrane vesicles and activation of numerous mechanisms dedicated to suppress dsRNA-dependent IFN induction [48]. This explains how these viruses employ mechanisms to escape, dampen, or block the antiviral interferon response in human cells. Furthermore, the ability of certain pathogens to escape from host immune response has been reported to be accomplished by host protein mimicry [49]. Thereby, no or a limited amount of antibodies are created, which also explains why certain vaccines may be ineffective. On the other hand, the similarity between pathogenic antigens and host proteins may lead to immune cross reactivity, whereby the reaction of the immune system toward the pathogenic antigens may harm similar human proteins, eventually causing autoimmune disease [50].

It has been proposed that an escape mechanism of SARS-CoV could be accomplished by viral expression of amino acid sequences mimicking ACTH. When the host produces antibodies against these viral antigens, the antibodies also bind to ACTH thereby limiting HPA activity and secretion of corticosteroids, which could lead to adrenal insufficiency [51]. This suggests that corticosteroids may be used to treat COVID-19 patients thereby helping the immune system to fight the infection. Indeed, corticosteroid supplements have improved the clinical conditions of many SARS patients [52]. On the other hand, a number of studies reported corticosteroid-treatment of SARS patients to be rather harmful (reviewed in [53]). For example, early steroid treatment has been associated with delayed viral clearance [54], and it has been found that patients with psychosis received higher cumulative doses of steroids than patients without psychosis [55]. In a study with 138 COVID-19 patients, where $45 \%$ received methylprednisolone, no effective outcome was observed after the treatment [56]. Thereby, the use of corticosteroids for the treatment of COVID-19 is not recommended [34].

\section{SARS-CoV-2 infection and psychological stress}

As mentioned above an infection with SARS-CoV-2 can be expected to lead to activation of the endocrine stress axis in association with mental stress. In addition, to the actual infection and treatment, psychological stress is likely to occur due to pandemic restrictions. Isolation during a pandemic is somewhat similar to physical immobilization 
stress, which leads to increased expression of enzymes involved in steroidogenesis [57] and elevated plasma corticosteroids [58]. Chronic restraint stress in rats also alters the RAAS by reduction of plasma aldosterone levels despite significant increases in plasma renin activity. Public anxiety of being infected is high; health care workers, who have higher vulnerability of acquiring and spreading the virus, are in particular those most likely to be distressed. In a study about the impact of MERS in health care workers in Saudi Arabia, a significant proportion expressed anxiety about the risk of acquiring MERS-CoV infection and for transmitting it to family members $[59,60]$.

In addition, during quarantine or curfew, people might experience loneliness because of the lack of communication and engagement with others. Loneliness, defined as the discrepancy between a person's desired and actual social relationships, is an emotional response to social isolation, while social isolation is an objective measure of the lack of social connections or interactions [61, 62]. Social isolation and loneliness are common sources of chronic stress in adults. They are correlated to a higher risk of mortality and cardiovascular disease, which have been associated with activation of the HPA axis and the sympathetic nervous system. Repeated and chronic social stress leads to glucocorticoid release, enhanced myelopoiesis, upregulated proinflammatory gene expression, and oxidative stress [63]. Furthermore, psychological stress due to social isolation is known to cause abnormal feeding behaviors. In addition to psychological reactions, such as depression and anxiety, stress triggers various physiological reactions including an increase in respiration and blood pressure. An activated stress axis also influences the body's metabolism [64, 65]. In particular, changes in feeding behavior are a well-known phenotype related to stress [66].

People who survived an infection with SARS-CoV have shown elevated stress levels long after the outbreak [67]. Leow et al. observed a direct effect on the HPA axis in SARS survivors 3 months after their recovery, where hypocortisolism was diagnosed. One year after recovery, HPA dysfunction had largely recovered [68]. However, even though physical conditions continuously improved, mental health did not. Psychiatric morbidities and chronic fatigue persisted and continued to be clinically significant up to at least 4 years after the SARS outbreak [69]. This has also been shown by Lee et al., where health care workers during the outbreak showed an elevated stress level when compared to non-health care workers. One year later, stress levels of survivors remained persistently elevated and higher than in control subjects. Specifically, health care workers showed significantly higher stress levels and had even higher depression, anxiety, and post-traumatic symptom scores than other survivors [70, 71].

\section{The role of stem cells in COVID-19}

Progenitor and stem cell populations are required for the successful homeostasis and adaptation of most tissues. Stem cells are dynamically regulated by signals originating from their niches, helping to regulate appropriate proliferation and differentiation. External and internal stressors influence the process of cell differentiation of stem and progenitor cells in the HPA axis in order to form the fully functional endocrine stress system [72].

In relation to coronavirus infections, it was shown that pulmonary stem/progenitor cells that express ACE2 are targeted by SARS-CoV in primary cultures [73]. The infected cells support active virus replication, which leads to their own destruction [73]. Another study by Mallick et al. has further revealed that a subset of these stem cells, the bronchoalveolar stem cells, are the prime target of the SARS-CoV infection of stem cells [74]. These observations suggest that, in addition to pneumocytes, lung stem cells/ progenitors are involved in coronavirus infection. This might also explain the long course of illness, in the context of continued deterioration of lung tissues and apparent loss of capacity of lung repair observed in COVID-19 patients.

As, after the lung, the adrenal is one of the most prominent organs infected by coronavirus. It would be reasonable to hypothesize that in a manner analogous to what happens in the lung, stem cells of the HPA axis could be affected by SARS-CoV-2. We and others have shown that stem cells play an important role in stress [75, 76]. Our work on the adrenal suggests that stem cell populations in both the adrenal cortex and medulla are highly susceptible to stress $[77,78]$. Under normal conditions, they seem to be quiescent or only slowly contribute to organ homeostasis. However, in stressful situations they enter the cell cycle leading to differentiation into mature lineages. Furthermore, we have shown that differentiation of adrenocortical stem cells is faster in females than in males [79].

In a recent paper, de Laval et al. have shown that a transient immune challenge with lipopolysaccharide not only induced an acute response in hematopoietic stem cells but also established persisting epigenetic modifications in myeloid lineage and innate immunity genes [80]. These results prove that permanent epigenetic changes are induced not only in the adaptive immune system but also in stem cells of the innate immune system after an infection.

\section{Conclusions and perspectives}

A pandemic such as the ongoing SARS-COV-2 outbreak is extremely stressful for all members of society, with longterm outcomes, not only for those who have been actually infected, but also for other people, who are not necessarily 
infected themselves, but who may suffer lasting consequences as a result of loss, grief, isolation, deprivation, and other psychological outcomes of ongoing quarantine or shelter in place measures. This includes health care workers, and others whose lives were severely affected.

The impact of multiple supportive and experimental treatments also needs to be considered. Many of these affect the endocrine stress axis, which again might lead to longterm consequences. From earlier coronavirus outbreaks, it is evident that survivors and health care workers are at increased risk of developing mental illness years after the outbreak. Such long-lasting impacts might be due to morphological changes in the brain and in the organs of the HPA axis. They could also be influenced by permanent epigenetic changes in stem cells as induced by the actual infection or the different forms of severe stress. Furthermore, sex differences in response to stress should be considered. All together these mechanisms could contribute to the increased risk of developing mental disorders after a pandemic such as COVID-19. Because of this enhanced long-term susceptibility to mental illness, it is imperative to monitor affected populations for an extended time during the course of, as well as after, the current and devastating COVID-19 pandemic.

Acknowledgements This work was supported by the Deutsche Forschungsgemeinschaft (DFG, German Research foundation) project no. 314061271, TRR 205/1: "The Adrenal: Central Relay in Health and Disease" and project no. 288034826, IRTG 2251: "Immunological and Cellular Strategies in Metabolic Disease".

\section{Compliance with ethical standards}

Conflict of interest The authors declare that they have no conflict of interest.

Publisher's note Springer Nature remains neutral with regard to jurisdictional claims in published maps and institutional affiliations.

\section{References}

1. Cameron HA, Schoenfeld TJ. Behavioral and structural adaptations to stress. Front Neuroendocrinol. 2018;49:106-13.

2. Licinio J, Wong ML. The role of inflammatory mediators in the biology of major depression: central nervous system cytokines modulate the biological substrate of depressive symptoms, regulate stress-responsive systems, and contribute to neurotoxicity and neuroprotection. Mol Psychiatry. 1999;4:317-27.

3. Nemeroff CB, Goldschmidt-Clermont PJ. Heartache and heartbreak-the link between depression and cardiovascular disease. Nat Rev Cardiol. 2012;9:526-39.

4. Reiche EM, Nunes SO, Morimoto HK. Stress, depression, the immune system, and cancer. Lancet Oncol. 2004;5:617-25.

5. Sternberg EM, Chrousos GP, Wilder RL, Gold PW. The stress response and the regulation of inflammatory disease. Ann Intern Med. 1992;117:854-66.
6. Zhang $\mathrm{H}$, Penninger JM, Li Y, Zhong N, Slutsky AS. Angiotensin-converting enzyme 2 (ACE2) as a SARS-CoV-2 receptor: molecular mechanisms and potential therapeutic target. Intensive Care Med. 2020;46:586-90.

7. Danser AHJ, Epstein M, Batlle D. Renin-Angiotensin System blockers and the COVID-19 pandemic: at present there is no evidence to abandon Renin-Angiotensin System blockers. Hypertension. 2020;75.

8. Patel AB, Verma A. COVID-19 and angiotensin-converting enzyme inhibitors and angiotensin receptor blockers: what is the evidence? JAMA. 2020.

9. Shang L, Zhao J, Hu Y, Du R, Cao B. On the use of corticosteroids for 2019-nCoV pneumonia. Lancet. 2020;395:683-4.

10. Vaduganathan M, Vardeny O, Michel T, McMurray JJV, Pfeffer MA, Solomon SD. Renin-angiotensin-aldosterone system inhibitors in patients with Covid-19. N Engl J Med. 2020;382:1653-9.

11. Xiang YT, Yang Y, Li W, Zhang L, Zhang Q, Cheung T, et al. Timely mental health care for the 2019 novel coronavirus outbreak is urgently needed. Lancet Psychiatry. 2020;7:228-9.

12. Agorastos A, Pervanidou P, Chrousos GP, Baker DG. Developmental trajectories of early life stress and trauma: a narrative review on neurobiological aspects beyond stress system dysregulation. Front Psychiatry. 2019;10:118.

13. Berger I, Werdermann M, Bornstein SR, Steenblock C. The adrenal gland in stress - adaptation on a cellular level. J Steroid Biochem Mol Biol. 2019;190:198-206.

14. Oyola MG, Handa RJ. Hypothalamic-pituitary-adrenal and hypothalamic-pituitary-gonadal axes: sex differences in regulation of stress responsivity. Stress. 2017;20:476-94.

15. Bale TL, Epperson CN. Sex differences and stress across the lifespan. Nat Neurosci. 2015;18:1413-20.

16. Hoffmann M, Kleine-Weber H, Schroeder S, Kruger N, Herrler T, Erichsen S, et al. SARS-CoV-2 cell entry depends on ACE2 and TMPRSS2 and is blocked by a clinically proven protease inhibitor. Cell. 2020;181:271-80 e278.

17. Walls AC, Park YJ, Tortorici MA, Wall A, McGuire AT, Veesler D. Structure, function, and antigenicity of the SARS-CoV-2 spike glycoprotein. Cell. 2020;181:281-92 e286.

18. Zhang L, Shen FM, Chen F, Lin Z. Origin and evolution of the 2019 novel coronavirus. Clin Infect Dis. 2020:ciaa112.

19. Ames MK, Atkins CE, Pitt B. The renin-angiotensin-aldosterone system and its suppression. J Vet Intern Med. 2019;33:363-82.

20. Arendse LB, Danser AHJ, Poglitsch M, Touyz RM, Burnett JC Jr., Llorens-Cortes C, et al. Novel therapeutic approaches targeting the Renin-Angiotensin System and associated peptides in hypertension and heart failure. Pharmacol Rev. 2019;71:539-70.

21. Li XC, Zhang J, Zhuo JL. The vasoprotective axes of the reninangiotensin system: Physiological relevance and therapeutic implications in cardiovascular, hypertensive and kidney diseases. Pharm Res. 2017;125(Pt A):21-38.

22. Santos RAS, Sampaio WO, Alzamora AC, Motta-Santos D, Alenina N, Bader M, et al. The ACE2/Angiotensin-(1-7)/MAS axis of the Renin-Angiotensin System: focus on angiotensin-(1-7). Physiol Rev. 2018;98:505-53.

23. Sparks MA, Crowley SD, Gurley SB, Mirotsou M, Coffman TM. Classical Renin-Angiotensin system in kidney physiology. Compr Physiol. 2014;4:1201-28.

24. Fang L, Karakiulakis G, Roth M. Are patients with hypertension and diabetes mellitus at increased risk for COVID-19 infection? Lancet Respir Med. 2020;8:e21.

25. Lei C, Qian K, Li T, Zhang S, Fu W, Ding M, et al. Neutralization of SARS-CoV-2 spike pseudotyped virus by recombinant ACE2Ig. Nat Commun. 2020;11:2070.

26. Wang L, de Kloet AD, Pati D, Hiller H, Smith JA, Pioquinto DJ, et al. Increasing brain angiotensin converting enzyme 2 activity 
decreases anxiety-like behavior in male mice by activating central Mas receptors. Neuropharmacology. 2016;105:114-23.

27. Wang LA, de Kloet AD, Smeltzer MD, Cahill KM, Hiller H, Bruce EB, et al. Coupling corticotropin-releasing-hormone and angiotensin converting enzyme 2 dampens stress responsiveness in male mice. Neuropharmacology. 2018;133:85-93.

28. McCray PB Jr., Pewe L, Wohlford-Lenane C, Hickey M, Manzel $\mathrm{L}$, Shi L, et al. Lethal infection of K18-hACE2 mice infected with severe acute respiratory syndrome coronavirus. J Virol. 2007;81:813-21.

29. Netland J, Meyerholz DK, Moore S, Cassell M, Perlman S. Severe acute respiratory syndrome coronavirus infection causes neuronal death in the absence of encephalitis in mice transgenic for human ACE2. J Virol. 2008;82:7264-75.

30. Yang XH, Deng W, Tong Z, Liu YX, Zhang LF, Zhu H, et al. Mice transgenic for human angiotensin-converting enzyme 2 provide a model for SARS coronavirus infection. Comp Med. 2007;57:450-9.

31. Zou X, Chen K, Zou J, Han P, Hao J, Han Z Single-cell RNA-seq data analysis on the receptor ACE2 expression reveals the potential risk of different human organs vulnerable to $2019-\mathrm{nCoV}$ infection. Front Med. 2020.

32. Lv Q, Yang Q, Cui Y, Yang J, Wu G, Liu M, et al. Effects of Taurine on ACE, ACE2 and HSP70 expression of hypothalamicpituitary-adrenal axis in stress-induced hypertensive rats. Adv Exp Med Biol. 2017;975(Pt 2):871-86.

33. Ding Y, He L, Zhang Q, Huang Z, Che X, Hou J, et al. Organ distribution of severe acute respiratory syndrome (SARS) associated coronavirus (SARS-CoV) in SARS patients: implications for pathogenesis and virus transmission pathways. J Pathol. 2004;203:622-30.

34. Guo YR, Cao QD, Hong ZS, Tan YY, Chen SD, Jin HJ, et al. The origin, transmission and clinical therapies on coronavirus disease 2019 (COVID-19) outbreak - an update on the status. Mil Med Res. 2020;7:11.

35. Gu J, Gong E, Zhang B, Zheng J, Gao Z, Zhong Y, et al. Multiple organ infection and the pathogenesis of SARS. J Exp Med. 2005;202:415-24.

36. Li YC, Bai WZ, Hashikawa T. The neuroinvasive potential of SARS-CoV2 may play a role in the respiratory failure of COVID19 patients. J Med Virol. 2020;92:552-5.

37. Wenham C, Smith J, Morgan R, Gender, Group C-W. COVID-19: the gendered impacts of the outbreak. Lancet. 2020;395:846-8.

38. Remuzzi A, Remuzzi G. COVID-19 and Italy: what next? Lancet. 2020;395:1225-8.

39. Yi Y, Lagniton PNP, Ye S, Li E, Xu RH. COVID-19: what has been learned and to be learned about the novel coronavirus disease. Int J Biol Sci. 2020;16:1753-66.

40. Chen N, Zhou M, Dong X, Qu J, Gong F, Han Y, et al. Epidemiological and clinical characteristics of 99 cases of 2019 novel coronavirus pneumonia in Wuhan, China: a descriptive study. Lancet. 2020;395:507-13.

41. Liu S, Zhang M, Yang L, Li Y, Wang L, Huang Z, et al. Prevalence and patterns of tobacco smoking among Chinese adult men and women: findings of the 2010 national smoking survey. $\mathbf{J}$ Epidemiol Community Health. 2017;71:154-61.

42. Russell G, Lightman S. The human stress response. Nat Rev Endocrinol. 2019;15:525-34.

43. Marx C, Ehrhart-Bornstein M, Scherbaum WA, Bornstein SR. Regulation of adrenocortical function by cytokines-relevance for immune-endocrine interaction. Horm Metab Res. 1998;30:416-20.

44. Kanczkowski W, Sue M, Bornstein SR. Adrenal gland microenvironment and its involvement in the regulation of stressinduced hormone secretion during sepsis. Front Endocrinol. 2016;7:156
45. Silverman MN, Pearce BD, Biron CA, Miller AH. Immune modulation of the hypothalamic-pituitary-adrenal (HPA) axis during viral infection. Viral Immunol. 2005;18:41-78.

46. Huang C, Wang Y, Li X, Ren L, Zhao J, Hu Y, et al. Clinical features of patients infected with 2019 novel coronavirus in Wuhan, China. Lancet. 2020;395:497-506.

47. Bornstein SR, Dalan R, Hopkins D, Mingrone G, Boehm BO. Endocrine and metabolic link to coronavirus infection. Nat Rev Endocrinol. 2020.

48. Kindler E, Thiel V, Weber F. Interaction of SARS and MERS Coronaviruses with the antiviral interferon response. Adv Virus Res. 2016;96:219-43.

49. Berger G. Escape of pathogens from the host immune response by mutations and mimicry. Possible means to improve vaccine performance. Med Hypotheses. 2015;85:664-9.

50. Segal Y, Shoenfeld Y. Vaccine-induced autoimmunity: the role of molecular mimicry and immune crossreaction. Cell Mol Immunol. 2018;15:586-94.

51. Wheatland R. Molecular mimicry of ACTH in SARS-implications for corticosteroid treatment and prophylaxis. Med Hypotheses. 2004;63:855-62.

52. Ho JC, Ooi GC, Mok TY, Chan JW, Hung I, Lam B, et al. High-dose pulse versus nonpulse corticosteroid regimens in severe acute respiratory syndrome. Am J Respir Crit Care Med. 2003;168:1449-56.

53. Stockman LJ, Bellamy R, Garner P. SARS: systematic review of treatment effects. PLoS Med. 2006;3:e343.

54. Lee N, Allen Chan KC, Hui DS, Ng EK, Wu A, Chiu RW, et al. Effects of early corticosteroid treatment on plasma SARSassociated Coronavirus RNA concentrations in adult patients. J Clin Virol. 2004;31:304-9.

55. Lee DT, Wing YK, Leung HC, Sung JJ, Ng YK, Yiu GC, et al. Factors associated with psychosis among patients with severe acute respiratory syndrome: a case-control study. Clin Infect Dis. 2004:39:1247-9.

56. Wang $\mathrm{D}, \mathrm{Hu} \mathrm{B}, \mathrm{Hu} \mathrm{C}$, Zhu F, Liu X, Zhang J, et al. Clinical characteristics of 138 hospitalized patients With 2019 Novel Coronavirus-Infected Pneumonia in Wuhan, China. JAMA. 2020;323:1061-9.

57. Aguilera G, Kiss A, Lu A, Camacho C. Regulation of adrenal steroidogenesis during chronic stress. Endocr Res. 1996;22:433-43.

58. Depke M, Fusch G, Domanska G, Geffers R, Volker U, Schuett C, et al. Hypermetabolic syndrome as a consequence of repeated psychological stress in mice. Endocrinology. 2008;149:2714-23.

59. Alsubaie S, Hani Temsah M, Al-Eyadhy AA, Gossady I, Hasan GM, Al-Rabiaah A, et al. Middle East Respiratory Syndrome Coronavirus epidemic impact on healthcare workers' risk perceptions, work and personal lives. J Infect Dev Ctries. 2019;13:920-6.

60. Al-Rabiaah A, Temsah MH, Al-Eyadhy AA, Hasan GM, AlZamil F, Al-Subaie S, et al. Middle East Respiratory SyndromeCorona Virus (MERS-CoV) associated stress among medical students at a university teaching hospital in Saudi Arabia. J Infect Public Health. 2020;13:687-91.

61. Holt-Lunstad J, Smith TB. Loneliness and social isolation as risk factors for CVD: implications for evidence-based patient care and scientific inquiry. Heart. 2016;102:987-9.

62. Holt-Lunstad J, Smith TB, Baker M, Harris T, Stephenson D. Loneliness and social isolation as risk factors for mortality: a meta-analytic review. Perspect Psychol Sci. 2015;10:227-37.

63. Xia N, Li H. Loneliness, social isolation, and cardiovascular health. Antioxid Redox Signal. 2018;28:837-51.

64. Block JP, He Y, Zaslavsky AM, Ding L, Ayanian JZ. Psychosocial stress and change in weight among US adults. Am J Epidemiol. 2009;170:181-92.

65. Patterson ZR, Abizaid A. Stress induced obesity: lessons from rodent models of stress. Front Neurosci. 2013;7:130. 
66. Yamada C, Saegusa Y, Nahata M, Sadakane C, Hattori T, Takeda $\mathrm{H}$. Influence of aging and gender differences on feeding behavior and ghrelin-related factors during social isolation in mice. PLoS ONE. 2015;10:e0140094.

67. Chrousos GP, Kaltsas G. Post-SARS sickness syndrome manifestations and endocrinopathy: how, why, and so what? Clin Endocrinol. 2005;63:363-5.

68. Leow MK, Kwek DS, Ng AW, Ong KC, Kaw GJ, Lee LS. Hypocortisolism in survivors of severe acute respiratory syndrome (SARS). Clin Endocrinol. 2005;63:197-202.

69. Lam MH, Wing YK, Yu MW, Leung CM, Ma RC, Kong AP, et al. Mental morbidities and chronic fatigue in severe acute respiratory syndrome survivors: long-term follow-up. Arch Intern Med. 2009;169:2142-7.

70. Lee AM, Wong JG, McAlonan GM, Cheung V, Cheung C, Sham $\mathrm{PC}$, et al. Stress and psychological distress among SARS survivors 1 year after the outbreak. Can J Psychiatry. 2007;52:233-40.

71. McAlonan GM, Lee AM, Cheung V, Cheung C, Tsang KW, Sham PC, et al. Immediate and sustained psychological impact of an emerging infectious disease outbreak on health care workers. Can J Psychiatry. 2007;52:241-7.

72. Mariniello K, Ruiz-Babot G, McGaugh EC, Nicholson JG, Gualtieri A, Gaston-Massuet C, et al. Stem cells, self-renewal, and lineage commitment in the endocrine system. Front Endocrinol. 2019;10:772.

73. Ling TY, Kuo MD, Li CL, Yu AL, Huang YH, Wu TJ, et al. Identification of pulmonary Oct-4+ stem/progenitor cells and demonstration of their susceptibility to SARS coronavirus (SARS$\mathrm{CoV}$ ) infection in vitro. Proc Natl Acad Sci USA. 2006;103:9530-5.

74. Mallick B, Ghosh Z, Chakrabarti J. MicroRNome analysis unravels the molecular basis of SARS infection in bronchoalveolar stem cells. PLoS ONE. 2009;4:e7837.

75. Bornstein SR, Steenblock C, Chrousos GP, Schally AV, Beuschlein F, Kline G, et al. Stress-inducible-stem cells: a new view on endocrine, metabolic and mental disease? Mol Psychiatry. 2019;24:2-9.

76. Steglich A, Hickmann L, Linkermann A, Bornstein S, Hugo C, Todorov V. Beyond the paradigm: novel functions of reninproducing cells. Rev Physiol Biochem Pharmacol. (In Press, 2020).

77. Steenblock C, Rubin de Celis MF, Delgadillo Silva LF, Pawolski $\mathrm{V}$, Brennand $\mathrm{A}$, Werdermann $\mathrm{M}$, et al. Isolation and characterization of adrenocortical progenitors involved in the adaptation to stress. Proc Natl Acad Sci USA. 2018;115:12997-3002.

78. Rubin de Celis MF, Garcia-Martin R, Wittig D, Valencia GD, Enikolopov G, Funk RH, et al. Multipotent glia-like stem cells mediate stress adaptation. Stem Cells. 2015;33:2037-51.

79. Grabek A, Dolfi B, Klein B, Jian-Motamedi F, Chaboissier MC, Schedl A. The adult adrenal cortex undergoes rapid tissue renewal in a sex-specific manner. Cell Stem Cell. 2019;25:290-6.

80. de Laval B, Maurizio J, Kandalla PK, Brisou G, Simonnet L, Huber $\mathrm{C}$, et al. C/EBPbeta-dependent epigenetic memory induces trained immunity in hematopoietic stem cells. Cell Stem Cell. 2020;S1934-5909(20)30017-5. 